\title{
Efficiency of Various Approaches for Feature Extraction in Palm Recognition- A Review
}

\author{
K.Suhasini \\ PG Scholar \\ Department of IT \\ Bannari Amman Institute of \\ Technology
}

\author{
S.Sanjushree \\ PG Scholar \\ Department of IT \\ Bannari Amman Institute of \\ Technology
}

\author{
C.Palanisamy \\ Professor and Head \\ Department of IT \\ Bannari Amman Institute of \\ Technology
}

\begin{abstract}
Security plays a very important role in the day to day life. In order to avoid unauthorized access by individuals authentication is mandatory in various fields such as industry, government sectors, colleges, online banking, military, etc., The traditional methods such as passwords or ID cards are less secure and forgery is possible. To overcome this, it is efficient to use biometric authentication methods such as palm print, iris, face recognition which are not identical among individuals. A review on various palm print feature extraction techniques is presented in this paper.
\end{abstract}

\section{General Terms}

Pattern Recognition, Security, Palm Print.

\section{Keywords}

Biometrics, palm print, recognition, authentication.

\section{INTRODUCTION}

Traditionally, passwords or ID cards have been used for applications, such as border and airport security, attendance control, online banking etc. These types of identity recognition methods present serious disadvantages, as they become less secure in a world where security plays a very important role(e.g. identify theft, terrorism) [1]. The increasing need for improved and higher security system has been accompanied by a continuous research and commercial growth of biometric related technologies being expected that the global biometric market is to grow at an annual rate of more than 20\% through 2012 according to a new market research report [2].

The various security issues in the society can be solved by using the biometric identification which relies on the physiological or behavioral characteristics of an individual. The physiological characteristics are considered to be more reliable than the behavioral characteristics since the possibility of forging is very difficult. The image of the palm works as the identity of an individual. The image of palm is used for enrollment and verification purpose where samples have to be given by the user and the comparison of those templates to corresponding claimed identity is done respectively.

An automated palm print recognition system evaluated the results in terms of correct recognition rate and verification rate. Correct recognition rate is the percentage of people that can be identified by the system. Verification rate can be calculated by using False Acceptance Rate (FAR), False Rejection Rate (FRR), as well as Equal Error Rate (EER). For a biometric to work effectively, FAR and FRR must be as low as possible.
Enrollment

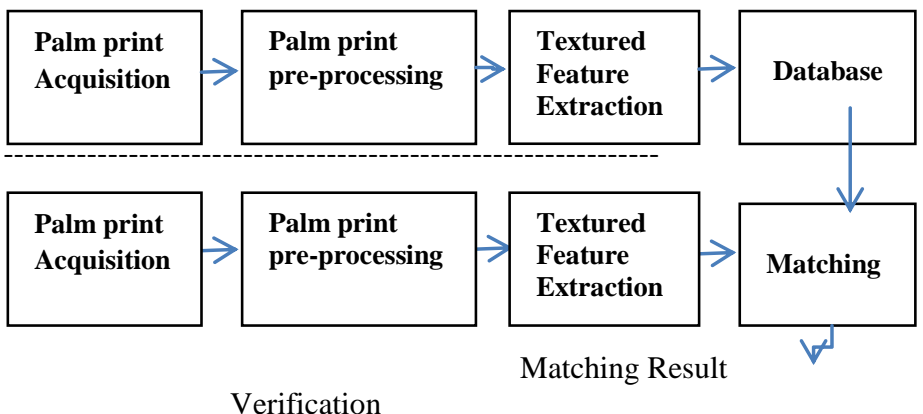

Fig 1: Block diagram of palm print verification system

The inner surface of a hand, its pattern of lines and shape of the surface, principle lines, wrinkles and creases are focused in a palm print. The inner surface mainly contains three flexion creases/ principle lines, secondary creases/wrinkles and ridges as shown in fig 2 [20]. The flexion and the major secondary creases are formed between $3^{\text {rd }}$ and $5^{\text {th }}$ months of pregnancy and superficial lines are formed after birth.

Palm print authentication can be divided into two categories, on-line and off-line[3]. On-line applications use low resolution images fig.2(b) (up to 75dpi) and off-line applications use high resolution images fig.2(a)(up to 500dpi).

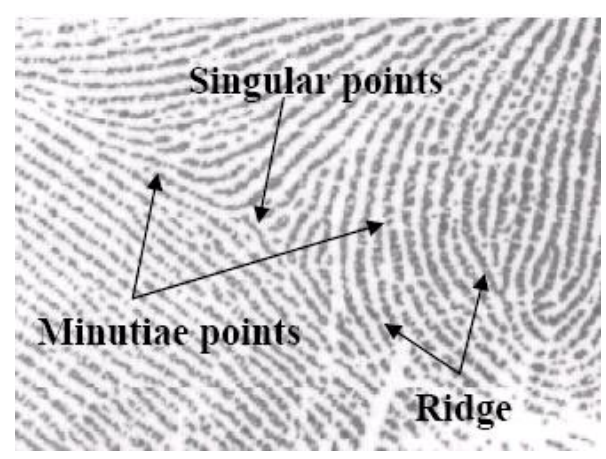

(a) 


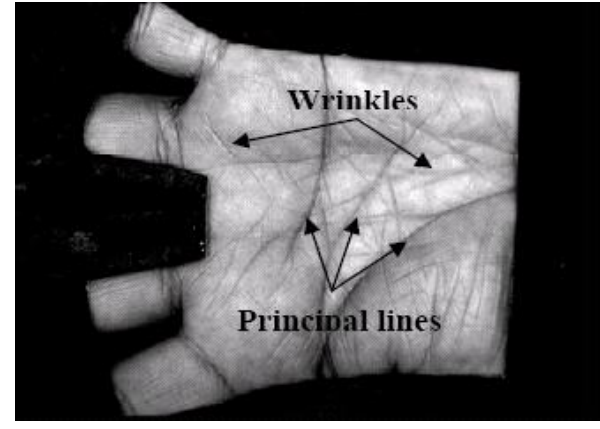

(b)

Fig 2: Palm print features in (a) a high resolution image and (b) a low resolution image.

\section{MODULES OF PALM PRINT RECOGNITION SYSTEM}

The four basic modules of palm print recognition system are palm print acquisition, pre-processing, feature extraction and matching. Each module plays an important role to enhance the performance of recognition system.

At the pre-processing stage the image is enhanced to remove noise and unwanted areas. Different pre-processing methods are explained in the coming sections. Feature extraction gets effective features from the pre-processed biometric trait. Feature extraction for palm print and fingerprint are different. After feature extraction fusion is carried out to combine different features and stored in the database as templates. A matching algorithm is used to compare it with the stored one in the database. Fig 3 gives the basic block diagram of a palm print identification system.

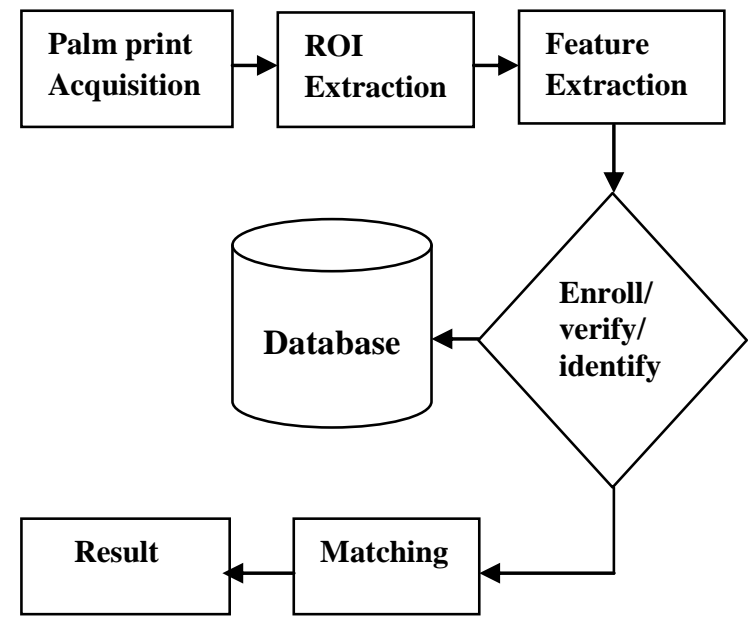

Fig 3: Palm print identification system

\subsection{Image Acquisition}

In this phase, image of palm print is first captured with the help of different types of digital cameras. A CCD-based scanner captures high resolution images and aligns palms accurately. The image acquired may be blurred and may contain noise. As a result the performance rate and quality of the palm print recognition system will be affected directly [13].So the image has to be pre-processed.

\subsection{Pre-Processing}

To reduce the overhead, instead of directly using the palm print images, preprocessing needs to be done. Preprocessing is used to remove distortion, align the palm prints and to crop the region of interest. This cropped ROI is used for feature extraction. The steps in pre-processing are as follows:

a. Binarize the palm image

b. Boundary tracking

c. Key points detection

d. Establishing a coordination system

e. Extracting the central part

\subsection{Feature Extraction}

Feature extraction is followed by pre-processing. In feature extraction phase features of palm like principal lines, orientation field, minutiae, density map, texture, singular points etc., are extracted.

The aim of this section is to recognize a correct person to authenticate and prevent unauthorized access. In identification, the system recognizes an individual by searching the templates of all users in the database for matching. Research on feature extraction and matching algorithms are classified as follows: Line based, subspace based, statistical based and coding based.

\subsubsection{Line Based Approaches}

This approach develops edge detectors or use existing one to extract palm lines [8]. Palm line are matched directly or represented in other format for matching. First using sobel masks magnitude of palm lines are computed. The magnitude are projected in $\mathrm{x}$ and $\mathrm{y}$ directions to form histograms. They computed first and second order derivative of palm images. These derivatives can be obtained by rotating the masks. The zero crossing of first order derivative is used to identify the edge points and corresponding directions. Second order derivative is used to identify the magnitude of the lines. In the feature vector the weighted sum of local directional magnitude is an element. Euclidian distance is used for matching [4].

Kung et al. formed a feature vector based on a low resolution edge map. The feature vector is passed into decision based neural networks [11].Han et al. used sobel and morphological operations to extract line like features from palm print images [5]

\subsubsection{Subspace Based Approaches}

Sub space based method is also called appearance based approach. This generally involves principal component analysis (PCA) [7], Linear discriminant analysis (LDA) and independent component analysis (ICA).The subspace coefficients are considered as features. In addition to applying PCA, LDA and ICA directly to palm print images, researchers also employ wavelets, Gabor, discrete cosine transform (DCT) and kernels in their methods [6], [9].

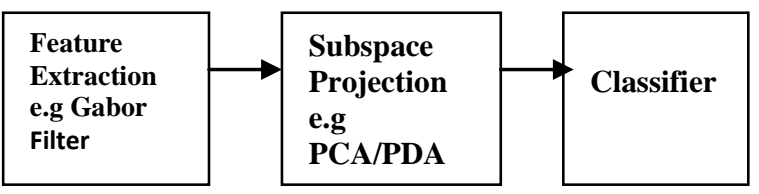

Fig 4: Architecture of subspace approach 2.3.3. Statistical Approaches

Statistical approaches are either local or global statistical approaches. Local statistical approaches transforms images into another domain and divide the transform into several 
regions such as mean and variance of each small regions[15]. Local statistics such as mean and variance of each small region are calculated and regarded as features. Global statistical approaches [14] compute global statistical features like moments, center of gravity and density directly from the whole transformed images. Moments, centers of gravity and density have been regarded as the global statistical features.

\subsubsection{Coding Approaches}

Palm code uses a single Gabor filter to extract the local phase information of palm print [16].Kong et al. introduced a fusion code method to encode the phase of the filter responses from a bank of Gabor filters with different orientations. A practical palm print recognition algorithm using $2 \mathrm{D}$ phase information (i) reduces the registered data size by registering quantized phase information and (ii) deals with nonlinear distortion between palm print images by local block matching using Phase-Only Correlation.

\subsubsection{Other Approaches}

Some approaches are difficult to classify because they combine several image processing methods to extract palm print features such as neural network to make final decision. Zhang et al. used complex wavelets to decompose palm print images and proposed a modified complex-wavelet structural similarity (CW-SSIM) index for measuring the local similarity of two images [17]. The overall similarity of two palm prints is estimated as the average of all local modified CW-SSIM. CW-SSIM is originally proposed for evaluating image quality [18]. Zhou et al. employ wavelet to decompose palm prints and use support vector machine (SVM) as a classifier. The input of the SVM is low sub-band images. This approach may overlook some important information in the middle frequency spectrum.

\subsubsection{Fusion}

Fusion is a promising approach that may increase the accuracy of systems. Fusion means combining other biometric traits with palm prints such as face, fingerprints, palm veins [22] and iris. Combining hand geometry and finger surface with palm print allows these features and palm print to be extracted from a single hand image i.e. only one sensor is needed. Researchers have examined fusion rules like sum, maximum, average, minimum, support vector machine (SVM) and neural networks. Researchers also fuse features including appearance-based, line and texture features from palm prints Fusion increases accuracy, computation costs and template sizes and reduces false acceptance[19]. Though Fusion increases computation costs and template sizes it reduces user acceptance.

\subsection{Matching}

Matching is next to the feature extraction phase. Feature matching determines the degree of similarity of recognition template with master template. Different approaches are used for matching. Input provided by individual is matched with templates present in database but it is difficult to identify effective features for the matching function. Matching can be carried out in three ways: hierarchical approach, classification and coding. Hierarchical approaches employ simple but computationally effective features to retrieve a subset of templates in a given database for further comparison [21]. These approaches increase matching speed at the cost of accuracy. Classification approaches assign a class to each biometric in a database. Many classification methods including KNN classifier [12], nearest neighbor classifier (1$\mathrm{NN}$ ) based on the Euclidean space [11] are being used widely. Coding approaches will use one matching function to search entire databases. This avoids errors from the classification or hierarchical systems. Hamming distance is used to find out the difference [20]. Table 1 specifies the accuracy of palm print recognition system using various features and classifier.

Table 1.Summary of palm print recognition

\begin{tabular}{|l|l|l|l|}
\hline Method & Feature & Classifier & Accuracy(\%) \\
\hline Gabor & $\begin{array}{l}\text { Phase } \\
\text { information }\end{array}$ & $\begin{array}{l}\text { Hamming } \\
\text { Distance }\end{array}$ & 97.59 \\
\hline PCA & Eigen Palm & L1 & 92.4 \\
\hline DCT & $\begin{array}{l}\text { Spectral } \\
\text { Features }\end{array}$ & $\begin{array}{l}\text { Distance } \\
\text { based }\end{array}$ & 99.97 \\
\hline DFT & $\begin{array}{l}\text { Statistic } \\
\text { Features }\end{array}$ & $\begin{array}{l}\text { Hamming } \\
\text { Distance }\end{array}$ & 95.48 \\
\hline Sobel & $\begin{array}{l}\text { Line } \\
\text { Feature }\end{array}$ & $\begin{array}{l}\text { Hamming } \\
\text { Distance }\end{array}$ & 94.84 \\
\hline FDA & Fisher Palm & L1 & 95.2 \\
\hline $\begin{array}{l}\text { Wavelet } \\
\text { Transfo } \\
\text { rm }\end{array}$ & $\begin{array}{l}\text { Wavelet } \\
\text { Energy }\end{array}$ & $\begin{array}{l}\text { Neural } \\
\text { network }\end{array}$ & 98 \\
\hline
\end{tabular}

\section{CONCLUSION}

In this paper we have reviewed the several existing methods used for palm print recognition system. This review shows the various approaches that are used for feature extraction in palm print identification. Instead of using the whole palm print image at a time, dominant spectral features have been extracted such as the major lines. The DCT method using spectral features which is based on distance based classifier provides more accuracy compared to other features extracted by various methods. This approach helps in increasing the performance and accuracy of the system. Some techniques are efficient and consume less time for identification process. The techniques can be selected based on the requirement.

\section{REFERENCES}

[1] A.Jain, A.Ross, and S.Prabhakar, "An introduction to biometric recognition," IEEE Trans. Circuits Systems Video Technol., vol. 14, no. 1, pp. 4-20, 2004.

[2] RNCOS, "Global Biometric Forecast to 2012", Market Research Report, 2008

[3] W.K. Kong, D. Zhang, Palm print texture analysis based on low-resolution images for personal authentication, in: proceedings of $16^{\text {th }}$ International Conference on Pattern Recognition, vol. 3, 2002, pp. 807-810.

[4] Z. Sun, T. Tan, Y. Wang, S.Z. Li, Ordinal palm print representation for personal identification, in: Proceeding of Computer Vision and Pattern Recognition, vol. 1, 2005, pp. 279-284.

[5] C.C. Han, A hand-based personal authentication using a coarse-to- fine strategy, Image and Vision Computing 22 (11) (2004) 909-918. 
[6] T. Connie, A.T.B. Jin, M.G.K. Ong, D.N.C. Ling, An automated palm print recognition system, Image and Vision Computing 23 (5) (2005) 501-515.

[7] Rafal Kozik and Michal Choras, "Combined shape and Texture information for palm print biometrics", Journal of Information Assurance and Security 5 (2010) 058-063

[8] X. Wu, D. Zhang, K. Wang, B. Huang, "Palm print classification using principal lines", Pattern Recognition 37 (10) (2004) 1987-1998.

[9] X. Wu, D. Zhang, K. Wang, Fisher palms based palm print recognition, Pattern Recognition Letters 24 (15) (2003) $2829-2838$

[10] G. Lu, D. Zhang, K. Wang, Palm print recognition using eigen palms features, Pattern Recognition Letters 24 (9) (2003) 1463-1467.

[11] Zhang, Y., Sun, D., Qiu, Z. 2010. Hand-based feature level fusion for single sample biometrics recognition. In Emerging Techniques and Challenges for Hand-Based Biometrics (ETCHB), 2010 International Workshop on (pp. 1-4). IEEE.

[12]Gayathri, R. Ramamoorthy, P. 2012. Fingerprint and palm print Recognition Approach based on Multiple Feature extraction. European Journal of scientific research. Vol 76 , No 4 .

[13] J. Dai and J. Zhou, "Multi feature-Based HighResolution Palm print Recognition," IEEE Transaction on Pattern Analysis and Machine Intelligence, Vol.33, No. 5, pp. 0162-8828, May 2011.

[14] L. Zhang, D. Zhang, "Characterization of palm prints by wavelet signatures via directional context modeling", IEEE Transactions on Systems, Man and Cybernetics, Part B, vol. 34, no. 3, pp. 1335-1347, 2004.
[15] J. You, W.K. Kong, D. Zhang, K.H. Cheung, "On hierarchical palm print coding with multiple features for personal identification in large databases", IEEE Transactions on Circuits and Systems for Video Technology, vol. 14, no. 2, pp. 234-243, 20

[16] D. Zhang, "Palm print authentication", nowell, mass kluwer academic publishers, 2004.

[17] L. Zhang, Z. Guo, Z. Wang and D. Zhang, "Palm print verification using complex wavelet transform", in Proceedings of International Conference on Image Processing, vol. 2, pp. 417- 420, 2007.

[18] Z. Wang, A.C. Bovik, H.R. Sheikh and E.P. Simoncelli, "Image quality assessment: From error to structural similarity", IEEE Trans. Image Processing, vol. 13, pp 600-612,2004.

[19] A. Kumar and D. Zhang, "Personal authentication using multiple palm print representation", Pattern Recognition, vol. 38, no. 10, pp. 1695-1704, 2005

[20]. K.Adams, Z. David, K. Mohamed, "A survey of palm print recognition", Pattern Recognition 42 (2009) 14081418.

[21] J. You, W.K. Kong, D. Zhang, K.H. Cheung, "On hierarchical palm print coding with multiple features for personal identification in large databases", IEEE Transactions on Circuits and Systems for Video Technology 14 (2) (2004) 234-243.

[22] S.Y. Kung, S.H. Lin, M. Fang, A neural network approach to face/palm recognition, in: Proceedings of IEEE Workshop Neural Networks for Signal Processing, 1995, pp. 323-332. 